\title{
AN ALGORITHM FOR SOLVING GENERALIZED ALGEBRAIC LYAPUNOV EQUATIONS IN HILBERT SPACE, APPLICATIONS TO BOUNDARY VALUE PROBLEMS
}

\author{
by LUCAS JÓDAR
}

(Received 10th June 1986)

\section{Introduction}

Let $L(H)$ be the algebra of all bounded linear operators on a separable complex Hilbert space $H$. In a recent paper [7], explicit expressions for solutions of a boundary value problem in the Hilbert space $H$, of the type

$$
\begin{gathered}
d / d t U(t)=A+B U(t)-U(t) C \\
E U(b)-U(0) F=G \\
0 \leqq t \leqq b
\end{gathered}
$$

are given in terms of solutions of an algebraic operator equation

$$
M U-U N=P
$$

If $H$ is finite-dimensional, an explicit algebraic expression for the solution of the equation (1.2) is given in [9]. For the infinite-dimensional case, and when the coefficient operators of (1.2) are annihilated by some analytic function, a methodology for solving the equation (1.2) is given in [6]. Sufficient conditions in order that (1.2) has a solution in certain classes of operators are given in [10] when $M$ and $P$ are selfadjoint operators. If $M$ is a right invertible operator and $N$ is a unilateral shift operator, a characterization is given in [1] of the operators $P$ for which the equation (1.2) is solvable.

If the Rosenblun's condition

$$
\sigma(M) \cap \sigma(N)=\emptyset
$$

is satisfied and $M$ and $N$ are infinite-dimensional unitary operators on $H$, the solution of (1.2) can be obtained by means of an infinite operator series. Under the uniqueness condition (1.3) and under certain conditions imposed on the operator coefficients, infinite integral expressions for the solution of the equation (1.2) are given in [2] and [5].

For $T$ in $L(H)$ we denote by $\sigma_{n}(T)$ the approximate point spectrum of $T$ and $\sigma_{\delta}(T)=$ $\{\lambda \in \mathbb{C} ; \lambda I-T$ is not onto $\}$. We denote by $\mathbb{C}_{*}$ the complementary set in the complex 
plane of the imaginary axis. Section 2 contains an algorithm for solving the equation (1.2) by application of a method of calculus of the square root of the identity operator in the Banach algebra $L(H)$. We apply the results to obtain explicit iterative approximations to the solutions of boundary value problems of the type (1.1).

\section{An algorithm for the algebraic operator equation $M U-U N=P$; Applications}

We begin with a theorem that allows us to obtain the operator $\operatorname{sig}(A)$ as the limit of a sequence of operators, where sig denotes the analytic function on $\mathbb{C}_{*}$, defined by $\operatorname{sig}(z)=1$, if the real part of $z, R z$ is positive and $\operatorname{sig}(z)=-1$, if $R z$ is negative. Notice that if $A$ lies in $L(H)$ and its spectrum $\sigma(A)$ is contained in the half plane $R z>0$, then from the minimal theorem, [4], one has $\operatorname{sig}(A)=I$. Analogously, if $\sigma(A)$ is contained in the half plane $R z<0$, then it follows that $\operatorname{sig}(A)=-I$.

Theorem 1. Let $A$ be an operator in $L(H)$ with spectrum $\sigma(A)$ contained in $\mathbb{C}_{*}$. Let $Z_{0}=A$, and let $\left\{Z_{n}\right\}_{n \geqq 0}$ be the sequence of operators defined by

$$
Z_{n+1}=\left(Z_{n}+Z_{n}^{-1}\right) / 2, \quad n \geqq 0 .
$$

Then the sequence $\left\{Z_{n}\right\}$ converges in the operator norm of $L(H)$ to $\operatorname{sig}(A)$.

Proof. First of all we show that $Z_{n}$ is an invertible operator in $L(H)$ for $n \geqq 0$. It is clear from the hypothesis that $Z_{0}=A$ is invertible. From the spectral mapping theorem, [4], the spectrum $\sigma\left(Z_{n+1}\right)$ is the set of all complex numbers of the type $(z+1 / z) / 2$, with $z$ belonging to $\sigma\left(Z_{n}\right)$. Moreover, it is easy to show that $R(z+1 / z)=\left(1+1 /|z|^{2}\right) R z$, for a non zero complex number $z$. Thus, $Z_{n+1}$ is invertible and $\operatorname{sig}(z+1 / z)=\operatorname{sig}(z)$, when $z$ lies in $\sigma\left(Z_{0}\right)$, in particular, it follows that $Z_{n}+\operatorname{sig}\left(Z_{0}\right)$ is an invertible operator in $L(H)$, for any $n \geqq 0$.

Let us consider the sequence of operators defined by the expression

$$
f_{n}(A)=\left(Z_{n}-\operatorname{sig}(A)\right)\left(Z_{n}+\operatorname{sig}(A)\right)^{-1}, \quad n \geqq 0 .
$$

Taking into account the commutativity between $Z_{n}$ and $\operatorname{sig}(A)$, and by computation it follows that

$$
\begin{aligned}
f_{n+1}(A) & =\left(\frac{Z_{n}^{2}+I}{2 Z_{n}}-\operatorname{sig}(A)\right)\left(\frac{Z_{n}^{2}+I}{2 Z_{n}}+\operatorname{sig}(A)\right)^{-1} \\
& =\left(\frac{Z_{n}^{2}+I-2 Z_{n} \operatorname{sig}(A)}{2 Z_{n}}\right)\left(\frac{Z_{n}^{2}+I+2 Z_{n} \operatorname{sig}(A)}{2 Z_{n}}\right)^{-1} \\
& =\left(Z_{n}^{2}-2 Z_{n} \operatorname{sig}(A)+I\right)\left(Z_{n}^{2}+2 Z_{n} \operatorname{sig}(A)+I\right)^{-1}=\left(Z_{n}-\operatorname{sig}(A)\right)^{2}\left(\left(Z_{n}+\operatorname{sig}(A)\right)^{2}\right)^{-1} \\
& =\left(\left(Z_{n}-\operatorname{sig}(A)\right)\left(Z_{n}+\operatorname{sig}(A)\right)^{-1}\right)^{2}=\left(f_{n}(A)\right)^{2} .
\end{aligned}
$$


From here, $f_{n}(A)=\left(f_{0}(A)\right)^{2 n}$, for $n \geqq 0$. If we prove that the spectral radius of the operator $f_{0}(A)$, denoted by $r\left(f_{0}(A)\right)$ is strictly less than one, then we will have

$$
\left\|f_{n}(A)\right\| \underset{n \rightarrow \infty}{\longrightarrow} 0, \text { in } L(H)
$$

From the spectral mapping theorem it follows that

$$
r\left(f_{0}(A)\right)=\sup \left\{|w| ; w \in \sigma\left(f_{0}(A)\right)\right\}=\sup \left\{\frac{|z-\operatorname{sig}(z)|}{|z+\operatorname{sig}(z)|} ; \quad z \in \sigma(A)\right\} .
$$

If $z$ lies in $\sigma(A)$, it is clear that $|z-\operatorname{sig}(z)|<|z+\operatorname{sig}(z)|$, thus, $r\left(f_{0}(A)\right) \leqq 1$.

If the spectral radius $r\left(f_{0}(A)\right)$ were one, then from the compactness of the spectrum $\sigma(A)$, there would be some $z_{0}$ in $\sigma(A)$ such that $\left|z_{0}-\operatorname{sig}\left(z_{0}\right)\right|=\left|z_{0}+\operatorname{sig}\left(z_{0}\right)\right|$. But it is not possible because in this case $R z_{0}=0$, in contradiction with the fact $z_{0} \in \sigma(A) \subset \mathbb{C}_{*}$. From the expression (2.2), the invertibility of $f_{n}(A)$, and by inversion it follows that

$$
Z_{n}=\left(I+f_{n}(A)\right)\left(I-f_{n}(A)\right)^{-1} \operatorname{sig}(A) .
$$

From (2.3) and (2.5), it follows that $\left\{Z_{n}\right\}_{n \geqq 0}$ converges to $\operatorname{sig}(A)$ in the operator norm of $L(H)$.

We denote by $\operatorname{sig}^{+}$the analytic function on $\mathbb{C}_{*}$, defined by $\operatorname{sig}^{+}(z)=1$, if $z \in \mathbb{C}_{*}$ and $R z>0$ and $\operatorname{sig}^{+}(z)=0$, if $z \in \mathbb{C}_{*}$ and $R z<0$, that is $\operatorname{sig}^{+}(z)=(1+\operatorname{sig}(z)) / 2$. The following results are based in a theorem of Davis-Rosenthal, which ensures the existence of solutions of the equation (1.2) when the spectral condition

$$
\sigma_{\delta}(M) \cap \sigma_{\pi}(N)=\emptyset
$$

is satisfied. Moreover, if $U$ is a solution of (1.2), the following similarity condition is verified

$$
\left[\begin{array}{cc}
M & -P \\
0 & N
\end{array}\right]=\left[\begin{array}{ll}
I & U \\
0 & I
\end{array}\right]\left[\begin{array}{ll}
M & 0 \\
0 & N
\end{array}\right]\left[\begin{array}{ll}
I & U \\
0 & I
\end{array}\right]^{-1}
$$

See [3] for details.

Suppose for a moment that $M$ and $N$ are operators such that that the condition (2.6) is satisfied and $\sigma(M) \subset \mathbb{C}_{*}, \sigma(N) \subset \mathbb{C}_{*}$. From (2.7) and the application of the RieszDunford functional calculus in (2.7) it follows that

$$
\begin{aligned}
\operatorname{sig}^{+}\left(\left[\begin{array}{cr}
M & -P \\
0 & N
\end{array}\right]\right) & =\left[\begin{array}{ll}
I & U \\
0 & I
\end{array}\right]\left(\operatorname{sig}^{+}\left(\left[\begin{array}{cc}
M & 0 \\
0 & N
\end{array}\right]\right)\right)\left[\begin{array}{cc}
I & -U \\
0 & I
\end{array}\right] \\
& =\left[\begin{array}{ll}
I & U \\
0 & I
\end{array}\right]\left[\begin{array}{cc}
\operatorname{sig}^{+}(M) & 0 \\
0 & \operatorname{sig}^{+}(N)
\end{array}\right]\left[\begin{array}{cc}
I & -U \\
0 & I
\end{array}\right] \\
& =\left[\begin{array}{cc}
\operatorname{sig}^{+}(M) & U \operatorname{sig}^{+}(N)-\operatorname{sig}^{+}(M) U \\
0 & \operatorname{sig}^{+}(N)
\end{array}\right] .
\end{aligned}
$$


With the hypothesis of Theorem 1 , if $\left\{Z_{n}\right\}_{n \geq 0}$ is the sequence of operators given in Theorem 1 , which converges to $\operatorname{sig}(A)$, then the sequence $\left(Z_{n}+I\right) / 2$, converges to $\operatorname{sig}^{+}(A)$, that is, $Z_{0}=A, Z_{n+1}=\left(Z_{n}+Z_{n}^{-1}\right) / 2$, satisfies

$$
\operatorname{sig}(A)=\lim _{n \rightarrow \infty} Z_{n} ; \quad \operatorname{sig}^{+}(A)=\lim _{n \rightarrow \infty}\left(Z_{n}+I\right) / 2 .
$$

Let $\left\{Z_{n}\right\}_{n \geqq 0}$ be the sequence of operators in $L(H \oplus H)$ which converges to the operator matrix

$$
A=\left[\begin{array}{rr}
M & -P \\
0 & N
\end{array}\right],
$$

then from Theorem 1, (2.9) and (2.6), it follows that

$$
\operatorname{sig}^{+}\left(\left[\begin{array}{cr}
M & -P \\
0 & N
\end{array}\right]\right)=\lim _{n \rightarrow \infty}\left(Z_{n}+Z_{n}^{-1}+2 I\right) / 4
$$

where

$$
\begin{aligned}
Z_{1} & =\left(Z_{0}+Z_{0}^{-1}\right) / 2=\left(\left[\begin{array}{cr}
M & -P \\
0 & N
\end{array}\right]+\left[\begin{array}{cr}
M & -P \\
0 & N
\end{array}\right]^{-1}\right) / 2 \\
& =(1 / 2)\left[\begin{array}{cc}
M+M^{-1} & M^{-1} P N-P \\
0 & N^{-1}
\end{array}\right]=\left[\begin{array}{cr}
M_{1} & -P_{1} \\
0 & N_{1}
\end{array}\right] .
\end{aligned}
$$

Recurrently one gets for $n>0$

$$
\begin{gathered}
Z_{n+1}=\left[\begin{array}{cr}
M_{n+1} & -P_{n+1} \\
0 & N_{n+1}
\end{array}\right]=(1 / 2)\left(\left[\begin{array}{lr}
M_{n} & -P_{n} \\
0 & N_{n}
\end{array}\right]+\left[\begin{array}{ll}
M_{n}^{-1} & M_{n}^{-1} P_{n} N_{n} \\
0 & N_{n}^{-1}
\end{array}\right]\right) \\
M_{n+1}=\left(M_{n}+M_{n}^{-1}\right) / 2 ; \quad N_{n+1}=\left(N_{n}+N_{n}^{-1}\right) / 2 \\
P_{n+1}=\left(P_{n}-M_{n}^{-1} P_{n} N_{n}\right) / 2 .
\end{gathered}
$$

From (2.8), (2.10) and (2.11) it follows that

$$
U \operatorname{sig}^{+}(N)-\operatorname{sig}^{+}(M) U=\lim _{n \rightarrow \infty} P_{n+1} / 2=\lim _{n \rightarrow \infty}\left(P_{n}-M_{n}^{-1} P_{n} N_{n}\right) / 4
$$

where $M_{0}=M, N_{0}=N$ and $P_{0}=P$. Notice that from (2.12) we can obtain an explicit expression of $U$ as limit of a sequence of operators, under certain conditions such as are stated in the following theorem.

For the operators $M$ and $N$ whose spectra are contained in $\mathbb{C}_{*}$, we can express their spectra as the union of two spectral sets. In fact, $\sigma(M)=\sigma_{1} \cup \sigma_{2}$, where $\sigma_{1}=$ 
$\{z \in \sigma(M) ; R z>0\} ; \sigma_{2}=\{z \in \sigma(M) ; R z<0\} ; \sigma(N)=\sigma_{3} \cup \sigma_{4}$, where $\sigma_{3}=\{z \in \sigma(N) ; R z>0\}$ $\sigma_{4}=\{z \in \sigma(N) ; R z<0\}$. Thus, one gets

$$
\operatorname{sig}^{+}(M)=E_{1}, \text { and } \operatorname{sig}^{+}(N)=E_{3}
$$

where $E_{1}$ and $E_{3}$ are the projection operators defined by means of the Riesz-Dunford functional calculus, [4],

$$
E_{1}=(1 / 2 \pi i) \int_{\gamma_{1}} e_{1}(z)(z I-M)^{-1} d z ; \quad E_{3}=(1 / 2 \pi i) \int_{\gamma_{3}} e_{3}(z)(z I-N)^{-1} d z
$$

where $\gamma_{i}$, for $i=1,3$ are appropriate Jordan curves containing $\sigma_{i}$ in their interior, and

$$
e_{1}(z)=\left\{\begin{array}{ll}
1, & z \in \sigma_{1} \\
0, & z \in \sigma(M) \sim \sigma_{1}
\end{array} ; \quad e_{3}(z)= \begin{cases}1, & z \in \sigma_{3} \\
0, & z \in \sigma(N) \sim \sigma_{3}\end{cases}\right.
$$

being

$$
E_{1}=e_{1}(M) ; \quad E_{3}=e_{3}(N)
$$

the operators obtained from the action of the Riesz-Dunford functional calculus on $M$ and $N$, respectively and the analytic functions $e_{i}(z)$, for $i=1,3$.

In particular, if $\sigma(M)$ is contained in $R z>0$, then $E_{1}=1$, and $E_{1}=0$ when $\sigma(M)$ is contained in $R z<0$.

Theorem 2. Let us consider the operator equation (1.2), where the spectra $\sigma(M)$ and $\sigma(N)$ are contained in $\mathbb{C}_{*}$ and satisfy the condition (2.7). Then:

(i) If $\sigma(M) \cap \sigma(N)=\emptyset$, and $\sigma(M)$ is contained in $R z<0, \sigma(N)$ is contained in $R z>0$ the only solution of (1.2) is given by

$$
U=\lim _{n \rightarrow \infty}\left(P_{n}-M_{n}^{-1} P_{n} N_{n}\right) / 4
$$

where $M_{n}, N_{n}$ and $P_{n}$, are given by (2.11) with $M_{0}=M, N_{0}=N$ and $P_{0}=P$.

(ii) If $\sigma(M)$ is contained in $R z>0$, and $\left(E_{3}-I\right)$ is an invertible operator in $L(H)$ then a solution of the equation (1.2) is given by

$$
U=\lim _{n \rightarrow \infty}\left(E_{3}-I\right)^{-1}\left(P_{n}-M_{n}^{-1} P_{n} N_{n}\right) / 4
$$

where $M_{n}, N_{n}$ and $P_{n}$, are given by (2.11) with $M_{0}=M, N_{0}=N$ and $P_{0}=P$.

(iii) If $\sigma(N) \cap \sigma(M)=\emptyset$, and $\sigma(M)$ is contained in $R z>0, \sigma(N)$ is contained in $R z<0$, the only solution of (1.2) is given by

$$
U=\lim _{n \rightarrow \infty}\left(M_{n}^{-1} P_{n} N_{n}-P_{n}\right) / 4
$$

where $M_{n}, N_{n}$ and $P_{n}$ are given as in (i). 
(iv) If $\sigma(N)$ is contained in $R z>0$, and $\left(I-E_{1}\right)$ is invertible operator in $L(H)$ then a solution of (1.2) is given by

$$
U=\lim _{n \rightarrow \infty}\left(P_{n}-M_{n}^{-1} P_{n} N_{n}\right)\left(I-E_{1}\right)^{-1 / 4}
$$

where $M_{n}, N_{n}$ and $P_{n}$, are given as in (ii).

Proof. It is a consequence of (2.12) and the previous remarks before the statement of the theorem.

The next theorem yields uniqueness and existence conditions for the boundary value problem (1.1) for the infinite-dimensional case and explicit approximations norm convergent in $L(H)$ to a solution of such problem.

Corollary 1. Let us consider the boundary value problem (1.1) and let $M, N$ and $P$ be the operators defined by

$$
\begin{gathered}
M=E \exp (b B) ; \quad N=F \exp (b C) \\
P=G \exp (b C)-E \int_{0}^{b} \exp ((b-s) B) A \exp (s C) d s
\end{gathered}
$$

then:

(i) The problem (1.1) is solvable if and only if the condition (2.6) is satisfied where $M, N$ and $P$ are given by (2.17).

(ii) The problem (1.1) has only one solution, if and only if, the condition (1.3) is satisfied.

(iii) Under the compatibility condition (i), a solution of (1.1) is given by the limit in the norm of $L(H)$ of the sequence

$$
V_{n}(t)=\exp (t B) U_{n} \exp (-t C)+\int_{0}^{t} \exp ((t-s) B) A \exp ((s-t) C) d s
$$

where $\left\{U_{n}\right\}_{n \geqq 0}$ is defined by the following expressions and under the following conditions:

(a) If $\sigma(N) \cap \sigma(M)=\emptyset, \sigma(M)$ is contained in $R z<0$, and $\sigma(N)$ is contained in $R z>0$, then

$$
U_{n}=\left(P_{n}-M_{n}^{-1} P_{n} N_{n}\right) / 4
$$

where $M_{n}, N_{n}$ and $P_{n}$ are given as in (2.11) with $M_{0}=M, N_{0}=N$ and $P_{0}=P$.

(b) If $\sigma(M)$ is contained in $R z>0$, and $\left(E_{3}-I\right)$ is invertible, then

$$
U_{n}=\left(E_{3}-I\right)^{-1}\left(P_{n}-M_{n}^{-1} P_{n} N_{n}\right) / 4
$$

where $M_{n}, N_{n}, P_{n}$ and $E_{3}$ are given as in (2.11) with $M_{0}=M, N_{0}=N, P_{0}=P$ and $E_{3}$ represents $\operatorname{sig}^{+}(N)$. 
Proof. (i) It is a consequence of Theorem 3(i) of [7], and Theorem 4 of [3]. (ii) It is Theorem 3(ii) of [7], and (iii) is a consequence of (i) and Theorem 2(i) and Theorem 2(ii).

Remark 1. Explicit expressions for the approximate solution $U_{n}$ of (1.2) may also be obtained under conditions on $M$ and $N$ analogous to those in Theorem 2(iii), (iv).

Acknowledgement. This research has been partially supported by a grant from the Ministerio de Educación y Ciencia de España.

\section{REFERENCES}

1. C. Apostol, On the operator equation $T X-X V=A$, Proc. Amer. Math. Soc. 59 (1976), 115118.

2. R. Bhatia, Сh. Davis and A. McIntosh, Perturbation of spectral subspaces and solution of linear operator equations, Linear Algebra Appl. 52/53 (1983), 45-67.

3. Ch. Davis and P. Rosenthal, Solving linear equations, Canad. J. Math. 26 (1974), 1384-1389.

4. N. Dunford and J. Schwartz, Linear Operators, Vols. I, II (Interscience, 1957).

5. E. Heinz, Beitrage zur Storungstheorie de Spektralzerlegung, Math. Ann. 123 (1951), 415-438.

6. V. Hernández and L. Jódar, Sobre la ecuación cuadrática en operadores $A+B T+T C+$ $T D T=0$, Stochastica 7 (1983).

7. L. JODAR, Boundary problems for Riccati and Lyapunov equations, Proc. Edinburgh Math. Soc. 29, (1986) 15-21.

8. H. Radjavi and P. Rosenthal, Invariant Subspaces (Springer, 1973).

9. A. JAMESON, Solution of the equation $A X+X B=C$ by inversion of an $M \times M$ matrix or an $N \times N$ matrix, SIAM J. Appl. Math. 16 (1968), 1020-1023.

10. J. A. Goldstein, On the operator equation $A X+X B=Q$, Proc. Amer. Math. Soc. 70 (1978).

Department of Applied Mathematics

Polytechnical University of Valencia

VALENCIA

SPAIN 